\title{
Positive Solutions for $(k, n-k)$ Conjugate Multipoint Boundary Value Problems in Banach Spaces
}

\author{
Yulin Zhao \\ School of Science, Hunan University of Technology, Zhuzhou 412007, China \\ Correspondence should be addressed to Yulin Zhao, zhaoylch@sina.com
}

Received 20 March 2012; Revised 20 May 2012; Accepted 3 June 2012

Academic Editor: Mohamed-Aziz Taoudi

Copyright (C) 2012 Yulin Zhao. This is an open access article distributed under the Creative Commons Attribution License, which permits unrestricted use, distribution, and reproduction in any medium, provided the original work is properly cited.

By means of the fixed point index theory of strict-set contraction operator, we study the existence of positive solutions for the multipoint singular boundary value problem $(-1)^{n-k} u^{(n)}(t)=f(t, u(t))$, $0<t<1, n \geq 2,1 \leq k \leq n-1, u(0)=\sum_{i=1}^{m-2} a_{i} u\left(\xi_{i}\right), u^{(i)}(0)=u^{(j)}(1)=\theta, 1 \leq i \leq k-1,0 \leq j \leq n-k-1$ in a real Banach space $E$, where $\theta$ is the zero element of $E, 0<\xi_{1}<\xi_{2}<\cdots<\xi_{m-2}<1, a_{i} \in[0,+\infty), i=$ $1,2, \ldots, m-2$. As an application, we give two examples to demonstrate our results.

\section{Introduction}

The theory of ordinary differential equations in Banach spaces has become a new important branch (see, e.g., [1-13] and the references cited therein). In 1988, Guo and Lakshmikantham [4] discussed multiple solutions for two-point boundary value problems of second-order ordinary differential equations in Banach spaces. In [7], Guo obtained the existence of positive solutions for a boundary value problem of $n$ th-order nonlinear impulsive integrodifferential equations in a Banach space by means of fixed point index theory and fixed point theory of completely continuous operators, respectively. Liu et al. in [6] obtained the existence of unbounded nonnegative solutions of a boundary value problem for $n$ th-order impulsive integrodifferential equations on an infinite interval in Banach spaces by means of the Mddotoch fixed point theory in a Banach space. Zhang et al. in [9] dealt with the existence, nonexistence, and multiplicity of positive solutions for a class of nonlinear threepoint boundary value problems of $n$ th-order differential equations in Banach spaces. Zhao and Chen in $[8,12]$ investigated the existence of at least triple positive solutions for nonlinear boundary value problem by upper and low solution methods. 
In this paper, the author considers the existence of positive solutions of the following higher-order $(k, n-k)$ conjugate multipoint boundary value problems (BVPs):

$$
\begin{gathered}
(-1)^{n-k} u^{(n)}(t)=f(t, u(t)), \quad t \in(0,1), n \geq 2,1 \leq k \leq n-1, \\
u(0)=\sum_{i=1}^{m-2} a_{i} u\left(\xi_{i}\right), \\
u^{(i)}(0)=u^{(j)}(1)=\theta, \quad 1 \leq i \leq k-1,0 \leq j \leq n-k-1
\end{gathered}
$$

in a real Banach space $E$, where $\theta$ is the zero element of $E, 0<\xi_{1}<\xi_{2}<\cdots<\xi_{m-2}<1, a_{i} \in$ $[0,+\infty), i=1,2, \ldots, m-2 . f:(0,1) \times E \rightarrow E$ is continuous and allowed to be singular at $t=0$ and $t=1$.

In scalar space, because of the widely applied background in mechanics and engineering, the nonlinear higher-order boundary value problems have received much attention (see Chyan and Henderson [Appl. Math. Letters 15 (2002) 767-774]). In [14], Eloe and Ahmad had solved successfully the existence of positive solution to the following $n$ th-order boundary value problems:

$$
\begin{gathered}
u^{(n)}(t)+a(t) f(u)=0, \quad t \in(0,1), \\
u^{(i)}(0)=0, \quad i=0,1, \ldots, n-2, \quad u(1)=a u(\eta) .
\end{gathered}
$$

Recently, the existence of solutions and positive solutions of nonlinear $(k, n-k)$ focal boundary value problem

$$
\begin{gathered}
(-1)^{n-k} u^{(n)}(t)=f\left(t, u(t), u^{\prime}(t), \ldots, u^{(n-1)}(t)\right), \quad t \in(0,1), \\
u^{(i)}(0)=0, \quad i=0,1, \ldots, k ; \quad u^{(j)}(1)=0, \quad j=k+1, k+2, \ldots, n-1,
\end{gathered}
$$

and its special cases has been studied by many authors (see, e.g., [15-23]).

By using the Krasnoselskii fixed point theorem, Eloe and Henderson in [15], Agarwal and $\mathrm{O}^{\prime}$ Regan in [16], and Kong and Wang in [20] have established the existence of solutions for following the $(k, n-k)$ conjugate boundary value problem:

$$
\begin{gathered}
(-1)^{n-k} u^{(n)}(t)=f(t, u(t)), \quad t \in(0,1), \\
u^{(i)}(0)=u^{(i)}(1)=0, \quad 0 \leq i \leq k-1,0 \leq j \leq n-k-1 .
\end{gathered}
$$

Very recently, by using the fixed point theory in a cone for strict-set contraction operators, Jiang and Zhang in [11] have discussed the existence of positive solutions for the above boundary value problem (1.4) in a Banach space, where the nonlinear term $f(t, u(t)): I \times E \rightarrow E$ is continuous but not allowed to have singularity at $t=0,1$, where $I=[0,1]$.

The organization of this paper is as follows. We shall introduce some lemmas and notations in the rest of this section. The preliminary lemmas are in Section 2. The main results 
are given in Section 3. Finally, two examples are presented to demonstrate our main results in Section 4.

Let the real Banach space $E$ with norm $\|\cdot\|$ be partially ordered by a cone $P$ of $E$, that is, $u \leq v$ if and only if $v-u \in P$, and $P^{*}$ denotes the dual cone of $P$, that is, $P^{*}=\{\varphi \mid \varphi \in$ $\left.E^{*}, \varphi(u) \geq 0, u \in P\right\}$. A cone is called a solid cone if the set of interior points is not empty.

The closed balls in spaces $E$ and $C[I, E]$ are denoted by $P_{r}=\{u \in E:\|u\| \leq r\}(r>0)$ and $B_{r}=\left\{u \in C[I, E]:\|u\|_{C} \leq r\right\}$, respectively.

The basic space used in this paper is $C[I, E]$. For any $u \in C[I, E]$, evidently, $\left(C[I, E],\|\cdot\|_{c}\right)$ is a Banach space with norm $\|u\|_{C}=\max _{t \in I}\|u(t)\|$, and $Q=\{u \in C[I, E]$ :

$u(t) \geq \theta$ for $t \in I\}$ is a cone of the Banach space $C[I, E]$. A function $u \in C^{n}[I, E]$ is called a positive solution of the boundary value problem (1.1) if it satisfies (1.1) and $u \in Q, u(t) \not \equiv \theta$.

Let $u(t): I \rightarrow E$ be continuous, and the abstract generalized integral $\int_{0}^{a} u(t) d t(a \in I)$ can be similarly defined as in the scalar spaces and $\int_{0}^{a} u(t) d t \in E$. If $\lim _{\varepsilon \rightarrow 0^{+}} \int_{0}^{\varepsilon} u(t) d t$ exist, then we say that the abstract integral is convergent, otherwise the abstract integral is divergent.

At the end of this section, we state some definitions and lemmas which will be used in Sections 2 and 3 (for details, see [1-3]).

Definition 1.1 (Kuratovski noncompactness measure). Let $E$ be a real Banach space, and $S$ is a bounded set in $E$. We denote $\alpha(S)=\inf \left\{\delta>0: S=\bigcup_{i=1}^{m} S_{i}\right.$, all the diameters of $\left.S_{i} \leq \delta\right\}$.

In the following, $\alpha(\cdot)$ denotes the Kuratowski measure of noncompactness in $E$ and $C[I, E]$.

Definition 1.2 (strict-set contraction operator). Let $E_{1}, E_{2}$ be real Banach spaces, and $S \subset E_{1}$. $T: S \rightarrow E_{2}$ is a continuous and bounded operator. If there exists a constant $k$, such that $\alpha(T(S)) \leq k \alpha(S)$, then $T$ is called a $k$-set contraction operator. When $k<1, T$ is called a strict-set contraction operator.

Lemma 1.3. If $D \subset C[I, E]$ is bounded and equicontinuous, then $\alpha(D(t))$ is continuous on $I$ and $\alpha(D)=\alpha(D(I))=\sup _{t \in I} \alpha(D(t))$, where $D(I)=\{u(t): u \in D, t \in I\}, D(t)=\{u(t): u \in D\}$.

Lemma 1.4. Let $P$ be a cone in a real Banach space $E$ and let $\Omega$ be a nonempty bounded open convex subset of $P$. Suppose that $T: \bar{\Omega} \rightarrow P$ is a strict-set contraction operator and $T(\bar{\Omega}) \subset \Omega$, where $\bar{\Omega}$ denotes the closure of in $P$. Then the fixed-point index $i(T, \Omega, P)=1$.

Lemma 1.5. Let $P$ be a cone in a real Banach space $E$ and let $\Omega$ be a bounded open subset of $P$, and suppose that $T: P \cap \bar{\Omega} \rightarrow P$ is a strict-set contraction operator.

(i) If $\theta \in \Omega$, and $T u \neq \lambda u$, for all $u \in \partial \Omega \cap P, \lambda \geq 1$. Then $i(T, \Omega \cap P, P)=1$.

(ii) If there exists $u_{0} \in P \backslash\{\theta\}$, such that $u-T u \neq \lambda u$, for all $u \in \partial \Omega \cap P, \lambda \geq 0$. Then $i(T, \Omega \cap P, P)=0$.

\section{The Preliminary Lemmas}

To prove the main results, we need the following lemmas. 
Lemma 2.1 (see [20]). Let $k(t, s)$ be the Green function for the $(k, n-k)$ conjugate BVP (1.4). Then

$$
k(t, s)=\frac{1}{(k-1) !(n-k-1) !} \begin{cases}\int_{0}^{t(1-s)} v^{k-1}(v+s-t)^{n-k-1} d v, & 0 \leq t \leq s \leq 1, \\ \int_{0}^{s(1-t)} v^{n-k-1}(v+t-s)^{k-1} d v, & 0 \leq s \leq t \leq 1 .\end{cases}
$$

Obviously, $k(t, s)$ is continuous on $I \times I$ and has the following properties.

$\left(G_{1}\right)$ There exist nonnegative functions $p(t), m(t), q(t) \in C[0,1]$ such that

$$
p(t) q(s) \leq k(t, s) \leq m(t) q(s) \leq q(s), \quad \forall t, s \in I,
$$

where

$$
p(t)=\frac{t^{k}(1-t)^{n-k}}{n-1}, \quad m(t)=\frac{t^{k-1}(1-t)^{n-k-1}}{\min \{k, n-k\}}, \quad q(s)=\frac{s^{n-k}(1-s)^{K}}{(k-1) !(n-k-1) !} .
$$

$\left(G_{2}\right)$ For any $\tau \in(0,1 / 2), k(t, s)$ satisfies

$$
k(t, s) \geq \rho(\tau) k(v, s), \quad t \in I_{\tau}=[\tau, 1-\tau], s, v \in I,
$$

where

$$
\rho(\tau):=\frac{\min _{t \in I_{\tau}} p(t)}{\max _{s \in[0,1]} m(s)} .
$$

Setting

$$
\Phi(t)=\frac{(n-1) !}{(k-1) !(n-k-1) !} \int_{t}^{1} s^{k-1}(1-s)^{n-k-1} d s .
$$

It is obvious that $0 \leq \Phi(t) \leq 1, t \in[0,1]$, and by the properties of the Euler integral, we have

$$
\Phi(0)=1, \quad \Phi(1)=0, \quad\|\Phi\|=1 .
$$

In order to abbreviate our discussion, we give the following assumptions.

$\left(C_{0}\right) \sum_{i=1}^{m-2} a_{i} \Phi\left(\xi_{i}\right)<1$.

$\left(C_{1}\right) f \in C[(0,1) \times P, P]$ and $\|f(t, u)\| \leq g(t)\|h(u)\|, t \in(0,1), u \in P$, where $h: P \rightarrow P$ is continuous and bounded and $g:(0,1) \rightarrow(0,+\infty)$ is continuous and satisfies $\int_{0}^{1} g(s) d s<+\infty$. 
$\left(C_{2}\right)$ For any $r>0$ and $[a, b] \subset(0,1), f(t, u)$ is uniformly continuous on $[a, b] \times P_{r}$.

$\left(C_{3}\right)$ There exists constant $L \geq 0$ such that for any $t \in(0,1)$ and the bounded set $D \subset P$

$$
\alpha(f(t, D)) \leq L \alpha(D)
$$

where

$$
2 L\left\{1+\left(1-\sum_{i=1}^{m-2} a_{i} \Phi\left(\xi_{i}\right)\right)^{-1} \sum_{i=1}^{m-2} a_{i}\right\} \cdot \max _{s \in I} q(s)<1
$$

Lemma 2.2. Let $\sum_{i=1}^{m-2} a_{i} \Phi\left(\xi_{i}\right) \neq 1$ and $\left(C_{1}\right)$ be satisfied, then the problem

$$
\begin{gathered}
(-1)^{n-k} u^{(n)}(t)=f(t, u(t)), \quad t \in I=[0,1], n \geq 2,1 \leq k \leq n-1, \\
u(0)=\sum_{i=1}^{m-2} a_{i} u\left(\xi_{i}\right), \\
u^{(i)}(0)=u^{(j)}(1)=\theta, \quad 1 \leq i \leq k-1,0 \leq j \leq n-k-1
\end{gathered}
$$

has a unique solution

$$
u(t)=\int_{0}^{1} K(t, s) f(s, u(s)) d s, \quad t \in I
$$

where

$$
K(t, s)=k(t, s)+\left(1-\sum_{i=1}^{m-2} a_{i} \Phi\left(\xi_{i}\right)\right)^{-1} \Phi(t) \sum_{i=1}^{m-2} a_{i} k\left(\xi_{i}, s\right), \quad 0 \leq t, s \leq 1
$$

Proof. According to the definitions of generalized integral in abstract space, the proof of this lemma is similar to the proof in scalar spaces, so we omit it.

For $u \in C[I, E]$, we define an operator $T$ by

$$
(T u)(t)=\int_{0}^{1} K(t, s) f(s, u(s)) d s, \quad t \in I
$$

Lemma 2.3. Suppose that $\left(C_{0}\right)-\left(C_{3}\right)$ hold. Then $T: C[I, E] \cap B_{r} \rightarrow C[I, E]$ is a strict-set contraction operator.

Proof. For $u \in u \in C[I, P]$, it follows from $\left(C_{1}\right)$ that $T$ is well defined and bounded operator. If $B \in C[I, P]$ is a bounded subset of $C[I, P]$, then $T B$ is bounded. 
Next we prove that $T$ is continuous on $C[I, E] \cap B_{r}$. Let $\left\{u_{j}\right\},\{u\} \subset C[I, E] \cap B_{r}$, and $\left\|u_{j}-u\right\|_{C} \rightarrow 0(j \rightarrow \infty)$. Hence $\left\{u_{j}\right\}$ is a bounded subset of $C[I, E] \cap B_{r}$. Thus, there exists $r>0$ such that $r=\sup _{j}\left\|u_{j}\right\|_{C}<\infty$ and $\|u\|_{C} \leq r$.

According to continuity of $f$, for all $\varepsilon>0$, there exists $J>0$ such that

$$
\left\|f\left(t, x_{j}(t)\right)-f(t, x(t))\right\|<\frac{\varepsilon}{\left[1+\left(1-\sum_{i=1}^{m-2} a_{i} \Phi\left(\xi_{i}\right)\right)^{-1} \sum_{i=1}^{m-2} a_{i}\right] \int_{0}^{1} q(s) d s},
$$

for $j \geq J$, for all $t \in I$.

Then,

$$
\begin{aligned}
\left\|\left(T u_{j}\right)(t)-(T u)(t)\right\| & \leq \int_{0}^{1} K(t, s)\left\|f\left(s, x_{j}(s)\right)-f(s,(s))\right\| d s \\
& \leq\left[1+\left(1-\sum_{i=1}^{m-2} a_{i} \Phi\left(\xi_{i}\right)\right)^{-1} \sum_{i=1}^{m-2} a_{i}\right] \int_{0}^{1} q(s) \| f\left(s, x_{j}(s)\right) \\
& -f(s, x(s)) \| d s<\varepsilon .
\end{aligned}
$$

Therefore, for all $\varepsilon>0$, for any $t \in I$ and $j \geq J$, we get

$$
\left\|\left(T u_{j}\right)(t)-(T u)(t)\right\| \longrightarrow 0
$$

This implies $T$ is continuous on $C[I, E] \cap B_{r}$. By the properties of continuity of $G(t, s)$, it is easy to see that $T$ is equicontinuous on $I$.

For any $S \subset C[I, E] \cap B_{r}$, it is easy to get that functions $T(S)=\{T u \mid u \in S\}$ are uniformly bounded. By Lemma 1.3, we get

$$
\alpha(T S)=\sup _{t \in I} \alpha((T S)(t)),
$$

where $(T S)(t)=\{(T u)(t): u \in S, t \in I$ is fixed $\}$.

Write

$$
\begin{gathered}
D=\left\{\int_{0}^{1} K(t, s) f(s, u(s)) d s: u \in S\right\}, \\
D_{\varepsilon}=\left\{\int_{\varepsilon}^{1-\varepsilon} K(t, s) f(s, u(s)) d s: u \in S\right\}, \quad\left(0<\varepsilon<\frac{1}{2}\right) .
\end{gathered}
$$


By $\left(C_{1}\right)$, for any $u \in S$, we have

$$
\begin{aligned}
H_{\varepsilon} & =\left\|\int_{\varepsilon}^{1-\varepsilon} K(t, s) f(s, u(s)) d s-\int_{0}^{1} K(t, s) f(s, u(s)) d s\right\| \\
& \leq c_{0} \int_{0}^{\varepsilon} K(t, s) g(s) d s+c_{0} \int_{1-\varepsilon}^{1} K(t, s) g(s) d s,
\end{aligned}
$$

where $c_{0}:=\max _{u \in B_{r}}\|h(u)\|$.

It follows from $H_{\varepsilon}$ and $\left(C_{1}\right)$ that the Hausdorff metric $d_{H}\left(D_{\varepsilon}, D\right) \rightarrow 0,\left(\varepsilon \rightarrow 0^{+}\right)$. Thus

$$
\lim _{\varepsilon \rightarrow 0^{+}} \alpha\left(D_{\varepsilon}\right)=\alpha(D)
$$

We next shall estimate $\alpha\left(D_{\varepsilon}\right)$. For any $u \in C[I, E]$, by $\int_{\mathcal{\varepsilon}}^{1-\varepsilon} u(t) d t \in(1-2 \varepsilon) \overline{c o}\{u(t): t \in$ $I\}$, then

$$
\begin{aligned}
\alpha\left(D_{\varepsilon}\right)= & \alpha\left(\left\{\int_{\varepsilon}^{1-\varepsilon} K(t, s) f(s, u(s)) d s: u \in S\right\}\right) \\
\leq & (1-2 \varepsilon) \alpha(\overline{c o}(\{K(t, s) f(s, u(s)): s \in[\varepsilon, 1-\varepsilon], u \in S\})) \\
\leq & \alpha(\{k(t, s) f(s, u(s)): s \in[\varepsilon, 1-\varepsilon], u \in S\}) \\
& +\left(1-\sum_{i=1}^{m-2} a_{i} \Phi\left(\xi_{i}\right)\right)^{-1} \alpha\left(\left\{\sum_{i=1}^{m-2} a_{i} k\left(\xi_{i}, s\right) f(s, u(s)): s \in[\varepsilon, 1-\varepsilon], u \in S\right\}\right) \\
\leq & \left(1+\left(1-\sum_{i=1}^{m-2} a_{i} \Phi\left(\xi_{i}\right)\right)^{-1} \sum_{i=1}^{m-2}\right) \cdot \max _{s \in I} q(s) \cdot \alpha\left(f\left(I_{\varepsilon} \times S\left(I_{\varepsilon}\right)\right)\right),
\end{aligned}
$$

where $I_{\varepsilon}=[\varepsilon, 1-\varepsilon], S\left(I_{\varepsilon}\right)=\left\{u(t): t \in I_{\varepsilon}\right\}$.

On the other hand, using a similar method as in the proof of Lemma 2 in [11] we can get that

$$
\alpha(S(I)) \leq 2 \alpha(S)
$$

Therefore, it follows from (2.19), (2.25) that

$$
\alpha\left(f\left(I_{\varepsilon} \times S\left(I_{\varepsilon}\right)\right)\right)=\sup _{t \in I_{\varepsilon}} \alpha\left(f\left(t, S\left(I_{\varepsilon}\right)\right)\right) \leq L \cdot \alpha\left(S\left(I_{\varepsilon}\right)\right) \leq L \cdot \alpha(S(I)) \leq 2 L \cdot \alpha(S) .
$$

Thus, we have

$$
\alpha\left(D_{\varepsilon}\right) \leq 2 L\left(1+\left(1-\sum_{i=1}^{m-2} a_{i} \Phi\left(\xi_{i}\right)\right)^{-1} \sum_{i=1}^{m-2} a_{i}\right) \cdot \max _{s \in I} q(s) \cdot \alpha(S) .
$$


Combining with (2.24), we get

$$
\alpha(D) \leq 2 L\left(1+\left(1-\sum_{i=1}^{m-2} a_{i} \Phi\left(\xi_{i}\right)\right)^{-1} \sum_{i=1}^{m-2} a_{i}\right) \cdot \max _{s \in I} q(s) \cdot \alpha(S)
$$

Therefore, we have

$$
\alpha(T S) \leq 2 L\left(1+\left(1-\sum_{i=1}^{m-2} a_{i} \Phi\left(\xi_{i}\right)\right)^{-1} \sum_{i=1}^{m-2} a_{i}\right) \cdot \max _{s \in I} q(s) \cdot \alpha(S), \quad \forall S \in P \cap B_{r} .
$$

Notice that by (2.9) we claim that $T: C[I, E] \cap B_{r} \rightarrow C[I, E]$ is a strict-set contraction. The proof is complete.

Further, we construct a cone $\Omega$ by

$$
\Omega=\left\{u \in Q: u(t) \geq \rho^{*}(\tau) u(s), \forall t \in I_{\tau}, \forall s \in I\right\},
$$

where $I_{\tau}=[\tau, 1-\tau]$, and

$$
\rho^{*}(\tau)=\min \left\{\rho(\tau), \min _{t \in I_{\tau}} \Phi(t)\right\}
$$

where $\rho(\tau)$ is defined in $\left(G_{2}\right)$. It is easy to see that $\Omega$ is a closed convex cone of $C[I, E]$ and $\Omega \subset Q$.

Lemma 2.4. Suppose that $\left(C_{0}\right)-\left(C_{3}\right)$ hold. Then, $T(\Omega) \subset \Omega$.

Proof. From $\left(G_{2}\right),(2.6),(2.15)$, and (2.30), for any $u \in \Omega, t \in T_{\tau}, t^{\prime} \in I$, we obtain

$$
\begin{aligned}
(T u)(t) & =\int_{0}^{1} K(t, s) f(s, u(s)) d s \\
& \geq \rho(\tau) \int_{0}^{1} k\left(t^{\prime}, s\right) f(s, u(s)) d s+\left(1-\sum_{i=1}^{m-2} a_{i} \Phi\left(\xi_{i}\right)\right)^{-1} \Phi(t) \int_{0}^{1} \sum_{i=1}^{m-2} a_{i} k\left(\xi_{i}, s\right) f(s, u(s)) d s \\
& \geq \rho^{*}(\tau)\left(\int_{0}^{1} k\left(t^{\prime}, s\right) f(s, u(s))\right) d s+\left(1-\sum_{i=1}^{m-2} a_{i} \Phi\left(\xi_{i}\right)\right)^{-1} \int_{0}^{1} \sum_{i=1}^{m-2} a_{i} k\left(\xi_{i}, s\right) f(s, u(s)) d s \\
& \geq \rho^{*}(\tau)\left[\int_{0}^{1} K\left(t^{\prime}, s\right) f(s, u(s)) d s\right]=\rho^{*}(\tau)(T u)\left(t^{\prime}\right), \quad t^{\prime} \in I .
\end{aligned}
$$

Therefore, $T(u) \in \Omega$, that is, $T(\Omega) \subset \Omega$. 


\section{The Main Results}

Let

$$
M^{*}:=\max _{s \in I} q(s) \cdot\left(1+\left(1-\sum_{i=1}^{m-2} a_{i} \Phi\left(\xi_{i}\right)\right)^{-1} \sum_{i=1}^{m-2} a_{i}\right) \int_{0}^{1} g(s) d s
$$

Theorem 3.1. Let $\left(C_{0}\right)-\left(C_{3}\right)$ hold. In addition, assume that the following conditions are satisfied:

$\left(C_{4}\right) d_{i}:=M^{*} c_{i}<1,(i=1,2)$, where

$$
c_{1}:=\varlimsup_{\|u\| \rightarrow \infty} \frac{\|h(u)\|}{\|u\|}, \quad c_{2}:=\varlimsup_{\|u\| \rightarrow \infty} \frac{\|h(u)\|}{\|u\|},
$$

$\left(C_{5}\right) P$ is a solid cone, and there exist $u_{0} \in \operatorname{Int}(P)$ and $\tau \in(0,1 / 2)$ such that $f(t, u) \geq$ $l(t) u_{0}, l(t) \in\left[I_{\tau}, R^{+}\right]$, and

$$
l_{0}=\min _{t \in I_{\tau}} p(t) \int_{\tau}^{1-\tau} q(s) l(s) d s \geq 1,
$$

for all $t \in I_{\tau}=[\tau, 1-\tau], u \geq u_{0}$.

Then problem (1.1) has at least two positive solutions $u_{1}, u_{2}$, and $u_{1}(t) \geq l_{0} u_{0}$, for $t \in I_{\tau}, u \geq$ $u_{0}$.

Proof. We first show that there exists $\beta>0$, such that

$$
\|v\| \geq \beta, \quad \text { for any } v \geq u_{0} .
$$

If (3.4) is not true, then there exist the sequences $\left\{v_{n}\right\}_{n=1}^{\infty}$ satisfying $v_{n} \geq u_{0}$, and $\left\|v_{n}\right\|<$ $1 / n,(n=1,2, \ldots)$. Thus $u_{0} \leq \theta$, which is contradiction with $u_{0} \in \operatorname{int}(P)$.

Let

$$
c_{i}^{\prime}=\frac{1+c_{i} M^{*}}{2 M^{*}}, \quad(i=1,2)
$$

Then

$$
c_{i}^{\prime}>c_{i}, \quad d_{i}^{\prime}=c_{i}^{\prime} M^{*}<1
$$

From $\left(C_{4}\right)$ and (3.6), there exist two positive constants $r_{1}, r_{2}$ with

$$
0<r_{1}<\beta, \quad r_{2}>\max \left\{\beta, 2\left\|u_{0}\right\|\right\}
$$

such that

$$
\|h(u)\| \leq c_{i}^{\prime}\|u\|, \quad u \in B_{r_{1}} \cap P, \quad\|h(u)\| \leq c_{i}^{\prime}\|u\|, \quad u \in P \backslash B_{r_{2}} .
$$


Therefore, for any $u \in P$, we have

$$
\|h(u)\| \leq c_{i}^{\prime}\|u\|+M
$$

where $M=\sup \left\{\|h(u)\|: u \in B_{r_{2}}\right\}$.

Choose

$$
r_{3}=r_{2}+\left(1-d_{2}^{\prime}\right)^{-1} M^{*} M
$$

In the following, let

$$
\begin{gathered}
\Omega_{1}=\left\{u \in Q: \mid\|u\|<r_{1}\right\}, \\
\Omega_{2}=\left\{\in Q: \mid\|u\|<r_{3}\right\}, \\
\Omega_{3}=\left\{u \in Q: \mid\|u\|<r_{3}, \text { if } t \in I_{\tau}, u(t) \geq u_{0}\right\} .
\end{gathered}
$$

It is to see from (3.7) that $\Omega_{3}$ is nonempty for $2 u_{0} \in \Omega_{3}$, which implies $\Omega_{3} \neq \emptyset$. Obviously, $\Omega_{i} \subset Q(i=1,2,3)$ are nonempty, convex, open sets and $\bar{\Omega}_{1}=Q \cap B_{r_{1}}, \bar{\Omega}_{2}=Q \cap B_{r_{2}}, \bar{\Omega}_{3}=\{u \in$ $\left.\bar{\Omega}_{2}: u(t) \geq u_{0}, t \in I_{\tau}\right\}$. So

$$
\Omega_{1} \subset \Omega_{2}, \quad \Omega_{3} \subset \Omega_{2}, \quad \Omega_{1} \cap \Omega_{3}=\emptyset
$$

For any $u \in \bar{\Omega}_{2}$, form (3.8) and (3.9), we have

$$
\begin{aligned}
\|(T u)(t)\| & \leq\left(\int_{0}^{1} K(t, s) g(s) d s\right)\left(c_{2}^{\prime}\|u\|+M\right) \\
& \leq \int_{0}^{1} g(s) d s \cdot\left(c_{2}^{\prime}\|u\|+M\right) \cdot\left(1+\left(1-\sum_{i=1}^{m-2} a_{i} \Phi\left(\xi_{i}\right)\right)^{-1} \sum_{i=1}^{m-2} a_{i}\right) \max _{s \in I} q(s) \\
& =d_{2}^{\prime}\|u\|+M^{*} M<d_{2}^{\prime} r_{3}+M^{*} M<r_{3}
\end{aligned}
$$

which implies

$$
T\left(\bar{\Omega}_{2}\right) \subset \Omega_{2}
$$

From (2.15) and (3.7), we get

$$
T\left(\bar{\Omega}_{1}\right) \subset \Omega_{1} .
$$


For any $u \in \bar{\Omega}_{3}$, it follows from (3.16) that $\|T u\|<r_{3}$. According to $\left(C_{5}\right)$ and $\left(G_{1}\right)$, we can obtain

$$
\begin{aligned}
(T u)(t) & \geq \int_{\tau}^{1-\tau} K(t, s) f(s, u(s)) d s \\
& \geq \int_{\tau}^{1-\tau} K(t, s) l(s) u_{0} d s \\
& \geq \min _{t \in I_{\tau}} p(t) \int_{\tau}^{1-\tau} q(s) l(s) d s \cdot u_{0}=l_{0} u_{0} \geq u_{0}, \quad \forall t \in I_{\tau},
\end{aligned}
$$

which implies

$$
T\left(\bar{\Omega}_{3}\right) \subset \Omega_{3}
$$

Combining (3.16)-(3.19) with Lemma 1.4, we have $i\left(T, \Omega_{i}, P\right)=1, i=1,2,3$. Furthermore, using the fixed point index theory, we obtain successively

$$
i\left(T, \Omega_{2} \backslash\left(\bar{\Omega}_{1} \cup \bar{\Omega}_{3}\right), P\right)=i\left(\mathrm{~T}, \Omega_{2}, P\right)-i\left(T, \Omega_{1}, P\right)-i\left(T, \Omega_{3}, P\right)=-1 .
$$

Then $T$ has at least two fixed points $u_{1}$ and $u_{2}$ which satisfy $u_{1} \in \Omega_{3}$ and $u_{2} \in \Omega_{2} \backslash$ $\left(\bar{\Omega}_{1} \cup \bar{\Omega}_{3}\right)$. Then Theorem 3.1 is proved.

Theorem 3.2. Assume that $\left(C_{0}\right)-\left(C_{3}\right), d_{2}<1$ hold. Suppose further that

$\left(C_{5}^{\prime}\right) P$ is a cone of the real Banach space $E$, and there exist $u_{0} \in P \backslash\{\theta\}$ and $\tau \in(0,1 / 2)$ such that $f(t, u) \geq l(t) u_{0}, l(t) \in\left[I_{\tau}, R^{+}\right]$, for for all $t \in I_{\tau}, u \geq u_{0}$.

Then problem (1.1) has at least one positive solutions $u$ with $u(t) \geq l_{0} u_{0}, t \in I_{\tau}, u \geq u_{0}$, where $l_{0}$ is defined in (3.3).

Proof. As in the proof of Theorem 3.1, we need only to show that $T$ has one positive fixed point $u$ with $u(t) \geq l_{0} u_{0}, t \in I_{\tau}, l_{0} \geq 1$.

Choose $r_{0}$ satisfying $r_{0}>\max \left\{\beta, 2\left\|u_{0}\right\|\right\}$ and let

$$
\Omega_{0}=\left\{u \in C[I, E]:\|u\|<r_{0}, u(t) \geq u_{0}, \forall t \in I_{\tau}\right\} .
$$

Obviously, $\Omega_{0}$ is a bounded closed convex set in $C[I, E] . \Omega_{0} \neq \emptyset$, for $u^{*}(t) \equiv u_{0} \in \Omega_{0}$. Let $u \in \Omega_{0}$. As the proof of (3.19), we have $T\left(\Omega_{0}\right) \subset \Omega_{0}$, where $T$ is given by (2.15). Thus, it follows from Lemmas 2.3 and 1.4 that $T$ has a fixed point $u \in \Omega_{0}$. The proof is complete.

Theorem 3.3. Assume that $\left(C_{0}\right)-\left(C_{3}\right)$ and the following conditions hold.

$\left(C_{6}\right)$ There exists $R_{0}>0$ such that $\sup _{P_{R_{0}}}\|h(u)\|<R_{0}\left(M^{*}\right)^{-1}$, where $P_{R_{0}}=\{u \in P$ : $\left.\|u\|<R_{0}\right\}$. 
$\left(C_{7}\right)$ There exists $\varphi \in P^{*}$ such that $\varphi(u)>0$ for any $u>\theta$, and

$$
\lim _{\|u\| \rightarrow+\infty} \min _{t \in I_{\tau}} \frac{\varphi(f(t, u))}{\varphi(u)}>M_{0}
$$

where $\tau \in(0,1 / 2)$, and

$$
M_{0}:=\left(\min _{t \in I_{\tau}} p(t) \cdot \rho^{*}(\tau) \int_{\tau}^{1-\tau} q(s) d s\right)^{-1}
$$

$\left(C_{8}\right)$ There exists $\varphi \in P^{*}$ such that $\varphi(u)>0$ for any $u>\theta$, and

$$
\lim _{\|u\| \rightarrow 0} \min _{t \in I_{\tau}} \frac{\varphi(f(t, u))}{\varphi(u)}>M_{0}
$$

where $\tau \in(0,1 / 2)$, and $M_{0}$ is defined in (3.23). Then problem (1.1) has at least two positive solutions.

Proof. If $\left(C_{6}\right)$ holds, then there exists $\varepsilon_{1}$ satisfying $0<\varepsilon_{1}<R_{0}\left(M^{*}\right)^{-1}$ such that $\|h(u)\| \leq$ $\left(R_{0}\left(M^{*}\right)^{-1}-\varepsilon_{1}\right)$. For any $u \in \Omega \cap \partial P_{R_{0}}, t \in I_{\tau}$, it follows from $\left(C_{1}\right)$ and (2.15) that

$$
\begin{aligned}
\|T u\| & \leq \int_{0}^{1} q(s) g(s)\|h(u)\| d s+\left(1-\sum_{i=1}^{m-2} a_{i} \Phi\left(\xi_{i}\right)\right)^{-1} \sum_{i=1}^{m-2} a_{i} \int_{0}^{1} q(s) g(s)\|h(u)\| d s \\
& \leq \max _{s \in I_{\tau}} q(s)\left(1+\left(1-\sum_{i=1}^{m-2} a_{i} \Phi\left(\xi_{i}\right)\right) \sum_{i=1}^{-1} a_{i}\right) \int_{0}^{1} g(s) d s \cdot\left(R_{0}\left(M^{*}\right)^{-1}-\varepsilon_{1}\right) \\
& =\left(R_{0}\left(M^{*}\right)^{-1}-\varepsilon_{1}\right) M^{*}<R_{0},
\end{aligned}
$$

which implies $T u \neq \lambda u$, for all $u \in \Omega \cap \partial P_{R_{0}}$, and $\lambda \geq 1$. From Lemma 1.5 (i), we have

$$
i\left(T, \Omega \cap \partial P_{R_{0}}, \Omega\right)=1 .
$$

According to $\left(C_{7}\right)$, there exist $\varepsilon_{2}>0$ and $R_{2}>R_{0}>0$, for any $t \in I_{\tau}$, such that

$$
\varphi(f(t, u)) \geq\left(M_{0}+\varepsilon_{2}\right) \varphi(u), \quad\|u\| \geq R_{2}
$$

Let $U_{2}=\left\{u \in C[I, E]:\|u\|<R_{2}\right\}$. We need only to show that $u-T u \neq \lambda$, for any $u \in \Omega \cap \partial U_{2}$, and $\lambda \geq 0, e \in P$ with $\|e\|=1$. If it is false, then there exists $u^{*} \in \Omega \cap \partial U_{2}$ and $\lambda_{0} \geq 0$, such that

$$
u^{*}-T u^{*}=\lambda_{0} e,
$$


which implies

$$
\begin{aligned}
u^{*}(t) & \geq T u^{*}(t)=\int_{0}^{1} K(t, s) f\left(s, u^{*}(s)\right) d s \\
& \geq p(t) \int_{\tau}^{1-\tau} q(s) f\left(s, u^{*}(s)\right) d s .
\end{aligned}
$$

Hence, for any $\varphi \in P^{*}$, we have

$$
\begin{aligned}
\varphi\left(u^{*}(t)\right) & \geq \varphi\left(T u^{*}(t)\right)=\int_{0}^{1} K(t, s) \varphi\left(f\left(s, u^{*}(s)\right)\right) d s \\
& \geq \int_{\tau}^{1-\tau} p(t) q(s)\left(M_{0}+\varepsilon_{2}\right) \varphi\left(u^{*}(s)\right) d s \\
& \geq \min _{t \in I_{\tau}} p(t) \int_{\tau}^{1-\tau} q(s)\left(M_{0}+\varepsilon_{2}\right) \varphi\left(u^{*}(s)\right) d s \\
& \geq\left(M_{0}+\varepsilon_{2}\right) \min _{t \in I_{\tau}} p(t) \cdot \rho^{*}(\tau) \int_{\tau}^{1-\tau} q(s) d s \cdot \varphi\left(u^{*}(t)\right) .
\end{aligned}
$$

It is easy to see that $\varphi\left(u^{*}(t)\right)>0$. In fact, if $\varphi\left(u^{*}(t)\right)=0$, since $u^{*} \in \Omega \cap \partial U_{2}$, then we have $\varphi\left(u^{*}(t)\right) \geq \rho^{*}(\tau) \varphi\left(u^{*}(s)\right) \geq 0$ and consequently $\left\|u^{*}\right\|=0$, which contradicts with $\left\|u^{*}\right\|=R_{2}$. By (3.29) and (3.30), we get $1<\left(M_{0}+\varepsilon_{2}\right) \min _{t \in I_{\tau}} p(t) \rho^{*}(\tau) \int_{\tau}^{1-\tau} q(s) d s \leq 1$, which is a contradiction. It follows from Lemma 1.5 (ii) that

$$
i\left(T, \Omega \cap \partial U_{2}, \Omega\right)=0 .
$$

If $\left(C_{8}\right)$ holds, then there exist $\varepsilon_{3}>0$ and $R_{1}>0$ with $R_{1}<R_{0}$, such that

$$
\varphi(f(t, u)) \geq\left(M_{0}+\varepsilon_{3}\right) \varphi(u), \quad\|u\| \geq R_{1}
$$

Let $U_{1}=\left\{u \in C[I, E]:\|u\|<R_{1}\right\}$. As in the proof of (3.31), for any $u \in \Omega \cap \partial U_{1}, t \in I_{\tau}$, we get

$$
i\left(T, \Omega \cap \partial U_{1}, \Omega\right)=0 .
$$

Notice that $\bar{U}_{1} \subset P_{R_{0}}, \bar{P}_{R_{0}} \subset U_{2}$. Thus, it follows from (3.26), (3.31), and (3.33) that

$$
\begin{aligned}
& \left(i\left(T, \Omega \cap\left(P_{R_{0}} \backslash \bar{U}_{1}\right)\right), \Omega\right)=i\left(T, \Omega \cap P_{R_{0}}, \Omega\right)-i\left(T, \Omega \cap U_{1}, \Omega\right)=1, \\
& \left(i\left(T, \Omega \cap\left(U_{2} \backslash \bar{P}_{R_{0}}\right)\right), \Omega\right)=i\left(T, \Omega \cap U_{2}, \Omega\right)-i\left(T, \Omega \cap P_{\mathrm{R}_{0}}, \Omega\right)=-1 .
\end{aligned}
$$

Then $T$ has at least two fixed points $u_{1}$ and $u_{2}$ which satisfy $u_{1} \in \Omega \cap\left(P_{R_{0}} \backslash \bar{U}_{1}\right)$ and $u_{2} \in \Omega \cap\left(U_{2} \backslash \bar{P}_{R_{0}}\right)$. Then Theorem 3.3 is proved. 
Similarly to the proofs of Theorem 3.3, we can easily get the following corollaries.

Corollary 3.4. Assume that $\left(C_{0}\right)-\left(C_{3}\right), d_{1}<1$ and $\left(C_{7}\right)$ hold. Then problem (1.1) has at least one positive solution.

Corollary 3.5. Assume that $\left(C_{0}\right)-\left(C_{3}\right), d_{2}<1$ and $\left(C_{8}\right)$ hold. Then problem (1.1) has at least one positive solution.

\section{Examples}

Now we present two examples to illustrate our main results.

Example 4.1. Consider the boundary value problems in $E=R^{N}(N$-dimensional Euclidean space and $\left.\|u\|=\max _{1 \leq p \leq N}\left|u_{p}\right|<+\infty\right)$

$$
\begin{gathered}
(-1)^{6} u_{p}^{(10)}(t)=\frac{1+t}{\sqrt{t(1-t)}}\left(\sin ^{2} u_{p+1}+\ln \left(1+u_{p}^{2}\right)+\sqrt{t(1-t)} H\left(u_{p}\right)\right), \quad 0<t<1, \\
u_{p}(0)=\frac{1}{9} u_{p}\left(\frac{1}{4}\right)+\frac{1}{2} u_{p}\left(\frac{1}{2}\right), \quad p=1,2,3, \ldots \\
u^{(i)}(0)=u^{(j)}(1)=0, \quad 1 \leq i \leq 3,0 \leq j \leq 5,
\end{gathered}
$$

where $n=10, k=4$, and

$$
H\left(u_{p}\right)= \begin{cases}\frac{2}{3} \times 10^{5} u_{p}+\frac{N}{\left\|u_{0}\right\|} \times 10^{12} u_{p}^{2}, & u_{p} \in\left[0, \frac{\left\|u_{0}\right\|}{N}\right], \\ \left(\frac{2}{3} \times 10^{5}+1 \times 10^{12}\right) u_{p}, & u_{p} \in\left[\frac{\left\|u_{0}\right\|}{N}, 1+r_{3}\left(\left\|u_{0}\right\|\right)+\frac{\left\|u_{0}\right\|}{N}\right], \\ \frac{2}{3} \times 10^{5} u_{p}+\sqrt{1+r_{3}\left(\left\|u_{0}\right\|\right)+\frac{\left\|u_{0}\right\|}{N}} & \\ \times 10^{12} \sqrt{u_{p}}, & u_{p} \in\left[1+r_{3}\left(\left\|u_{0}\right\|\right)+\frac{\left\|u_{0}\right\|}{N},+\infty\right),\end{cases}
$$

where $r_{3}(\cdot)$ is as defined in (3.10). Then the problem (4.1) can be regarded as a BVP of the form (1.1) in $E$. In this situation, $u=\left(u_{1}, \ldots, u_{P}, \ldots, u_{N}\right) \in R^{N}, f=\left(f_{1}, \ldots, f_{P}, \ldots, f_{N}\right), \theta=$ $(0,0, \ldots, 0) \in R^{N}$ and $a_{1}=1 / 9, \xi_{1}=1 / 4, a_{2}=\xi_{2}=1 / 2, g=\left(g_{1}, \ldots, g_{P}, \ldots, g_{N}\right), h=$ $\left(h_{1}, \ldots, h_{P}, \ldots, h_{N}\right)$, in which

$$
\begin{gathered}
f_{p}\left(t, u_{1}, u_{2}, \ldots, u_{N}\right)=\frac{1+t}{\sqrt{t(1-t)}}\left(\sin ^{2} u_{p+1}+\ln \left(1+u_{p}^{2}\right)+\sqrt{t(1-t)} H\left(u_{p}\right)\right), \\
p(t)=\frac{t^{4}(1-t)^{6}}{9}, \quad m(t)=\frac{t^{3}(1-t)^{5}}{4}, \quad q(s)=\frac{s^{6}(1-s)^{4}}{3 ! \cdot 5 !} .
\end{gathered}
$$


Obviously, $f:(0,1) \times E \rightarrow E$ is continuous. Taking $\tau=1 / 4$. By direct account, we have

$$
\sum_{i=1}^{m-2} a_{i} \Phi\left(\xi_{i}\right)=\frac{1}{9} \Phi\left(\frac{1}{4}\right)+\frac{1}{2} \Phi\left(\frac{1}{2}\right) \approx 0.219<1
$$

Now we show that $\left(C_{1}\right)-\left(C_{5}\right)$ hold.

First, it is easy to see that $\|f(t, u)\| \leq g(t)\|h(u)\|, t \in(0,1), u \in E$, with

$$
g_{p}(t)=\frac{2}{\sqrt{t(1-t)}}, \quad h_{p}(u)=\sin ^{2} u_{p+1}+\ln \left(1+u_{p}^{2}\right)+\frac{1}{2} H\left(u_{p}\right)
$$

and $\int_{0}^{1} g_{p}(s) d s=2 \pi<+\infty$

For sufficiently large number $R>0$, take $P=\left\{u=\left(u_{1}, \ldots, u_{p}, \ldots, u_{N}\right) \in R^{N}: u_{p} \geq\right.$ $0,\|u\| \leq R, p=1,2, \ldots, N\}$.

Then $h: P \rightarrow P$ is continuous and bounded. Therefore, $\left(C_{1}\right)$ is satisfied. In addition, $\left(C_{2}\right)$ and $\left(C_{3}\right)$ are automatically satisfied in $N$-dimensional Euclidean space $R^{N}$.

As far as $\left(C_{4}\right)$ is concerned, we get

$$
\begin{aligned}
& M^{*}=2 \pi \max _{s \in[0,1]} \frac{s^{6}(1-s)^{4}}{3 ! 5 !} \cdot\left(1+(1-0.219)^{-1} \times\left(\frac{1}{9}+\frac{1}{2}\right)\right) \approx 1.86 \times 10^{-5}, \\
& c_{1}=\varlimsup_{\|u\| \rightarrow \infty} \frac{\|h(u)\|}{\|u\|}=\frac{1}{3} \times 10^{5}, \quad c_{2}=\varlimsup_{\|u\| \rightarrow \infty} \frac{\|h(u)\|}{\|u\|}=\frac{1}{3} \times 10^{5}, \quad d_{i}=M^{*} c_{i} \approx 0.62<1 .
\end{aligned}
$$

Then $\left(C_{4}\right)$ is satisfied.

On the other hand, $P$ is a solid cone, for all $u_{0} \in \operatorname{Int}(P)$. For all $t \in I_{\tau}=[1 / 4,3 / 4]$, we have

$$
f_{p}(t, u) \geq 1 \times 10^{12}(1+t) u_{p} \geq l(t) u_{0}^{(p)}, \quad \text { for } u \geq u_{0}
$$

where $u_{0}=\left(u_{0}^{(1)}, \ldots, u_{0}^{(p)}, \ldots, u_{0}^{(N)}\right), l(t)=1 \times 10^{12}(1+t)$, and

$$
l_{0}=\min _{t \in[1 / 4,3 / 4]} p(t) \int_{1 / 4}^{3 / 4} q(s) l(s) d s \approx 7.055>1 .
$$

So $\left(C_{5}\right)$ is also satisfied. For example, if $u_{0}=\{1,1, \ldots, 1\}$, we let $\beta=1$, then $r_{3}\left(\left\|u_{0}\right\|\right)+$ $\left(\left\|u_{0}\right\|\right) / N+1 \approx 9.745 \times 10^{7}(2 N+1)$. By Theorem 3.1, the problem (4.1) has at least two positive solutions in $E$. 
Example 4.2. Consider the following boundary value problems in $l^{\infty}$ :

$$
\begin{gathered}
(-1)^{3} u_{p}^{(9)}(t)=\frac{\pi}{\sqrt{t}}\left(\ln \left(1+\frac{u_{p}^{2}}{p}\right)+\frac{t(1-t) \sqrt{u_{p}}}{p}\left|\sin u_{p+1}\right|+H\left(u_{p}\right)\right), \quad 0<t<1, \\
u_{p}(0)=\frac{1}{4} u_{p}\left(\frac{3}{4}\right), \quad p=1,2,3, \ldots \\
u^{(i)}(0)=u^{(j)}(1)=0, \quad 1 \leq i \leq 5,0 \leq j \leq 2,
\end{gathered}
$$

where $n=9, k=6$, and

$$
H\left(u_{p}\right)= \begin{cases}8 \times 10^{8} u_{p}, & u_{p} \in\left[0, \frac{1}{16} \times 10^{-8}\right], \\ 100 \sqrt[4]{u_{p}}, & u_{p} \in\left[\frac{1}{16} \times 10^{-8}, 1 \times 10^{4}\right], \\ 8 \times 10^{8} u_{p}+\left(10-8 \times 10^{10}\right) \sqrt{u_{p}}, & u_{p} \in\left[1 \times 10^{4},+\infty\right) .\end{cases}
$$

Let $P=\left\{u=\left(u_{1}, \ldots, u_{p}, \ldots\right) \in l^{\infty}: u_{p} \geq 0, p=1,2, \ldots\right\},\|u\|=\sup _{p \geq 1}\left|u_{p}\right|<+\infty$. Then $l^{\infty}$ is a Banach space. Thus the problem (4.9) can be regarded as a BVP of the form (1.1) in $l^{\infty}$. In this situation, $u=\left(u_{1}, \ldots, u_{P}, \ldots\right) \in l^{\infty}, f=\left(f_{1}, \ldots, f_{P}, \ldots\right), \theta=(0,0, \ldots) \in l^{\infty}$ and $a_{1}=1 / 4, \xi=3 / 4, g=\left(g_{1}, \ldots, g_{P}, \ldots,\right), h=\left(h_{1}, \ldots, h_{P}, \ldots,\right)$, in which

$$
\begin{gathered}
f_{p}\left(t, u_{1}, u_{2}, \ldots,\right)=\frac{\pi}{\sqrt{t}}\left(\ln \left(1+\frac{u_{p}^{2}}{p}\right)+\frac{t(1-t) \sqrt{u_{p}}}{p}\left|\sin u_{p+1}\right|+H\left(u_{p}\right)\right), \\
p(t)=\frac{t^{6}(1-t)^{3}}{8}, \quad m(t)=\frac{t^{5}(1-t)^{2}}{3}, \quad q(s)=\frac{s^{3}(1-s)^{6}}{5 ! \cdot 2 !} .
\end{gathered}
$$

Obviously, $f:(0,1) \times E \rightarrow E$ is continuous taking $\tau=1 / 8$. By direct account, we have

$$
\begin{gathered}
\sum_{i=1}^{m-2} a_{i} \Phi\left(\xi_{i}\right)=\frac{1}{4} \Phi\left(\frac{3}{4}\right) \approx 0.0804<1, \quad M^{*}=1.099 \times 10^{-4}, \\
\rho^{*}(\tau)=\min \left\{\rho(\tau), \min _{[1 / 8,7 / 8]} \Phi(t)\right\} \approx \min \{0.6471,0.0674\}=0.0674 .
\end{gathered}
$$


Let

$$
g_{p}(t)=\frac{\pi}{\sqrt{t}}, \quad h_{p}(u)=\ln \left(\left(1+\frac{u_{p}^{2}}{p}\right)+\frac{\sqrt{u_{p}}}{2 p}\left|\sin u_{p+1}\right|+H\left(u_{p}\right)\right)
$$

and $\int_{0}^{1} g_{p}(s) d s=2 \pi<+\infty$,

$$
M_{0}=\left(\min _{t \in[1 / 8,7 / 8]} p(t) \cdot \rho^{*}(\tau) \int_{1 / 8}^{7 / 8} q(s) d s\right)^{-1} \approx 7.0811 \times 10^{8} .
$$

Similar to the proofs of Example 4.1, we can show that $\left(C_{1}\right)-\left(C_{3}\right)$ hold. Taking $R_{0}=10^{8 / 3}$, then $\sup _{u \in P_{R_{0}}}\{\|h(u)\|\}<M^{*} R_{0}$, which implies $\left(C_{6}\right)$, holds.

For $u \in P$, choose $\varphi \in P^{*}$ with $\varphi(u)=\sum_{p=1}^{+\infty} u_{p} / p^{2}$. Obviously, $\varphi(u)>0$ for $u>\theta$, and

$$
\begin{aligned}
& \lim _{\|u\| \rightarrow+\infty} \min _{t \in[1 / 8,7 / 8]} \frac{\varphi(f(t, u))}{\varphi(u)} \\
& \quad=\lim _{\|u\| \rightarrow+\infty} \min _{t \in[1 / 8,7 / 8]} \frac{\pi}{\sqrt{t}} \cdot \frac{\sum_{p=1}^{+\infty} 1 / p^{2}\left\{\ln \left(1+u_{p}^{2} / p\right)+\sqrt{u_{p}} / 2 p\left|\sin u_{p+1}\right|+H\left(u_{p}\right)\right\}}{\sum_{p=1}^{+\infty} u_{p} / p^{2}} \\
& \quad>8 \times 10^{8}>M_{0},
\end{aligned}
$$

which implies $\left(C_{7}\right)$ is satisfied. Similarly, we can show that $\left(C_{8}\right)$ holds. By Theorem 3.3 , the problem (4.9) has at least two positive solutions in $E$.

\section{References}

[1] D. Guo, V. Lakshmikantham, and X. Liu, Nonlinear Integral Equations in Abstract Spaces, Kluwer Academic Publishers, Dordrecht, The Netherlands, 1996.

[2] K. Deimling, Ordinary Differential Equations in Banach Spaces, Springer, Berlin, Germany, 1977.

[3] V. Lakshmikantham and S. Leela, Nonlinear Differential Equations in Abstract Spaces, Pergamon Press, Oxford, UK, 1981.

[4] D. J. Guo and V. Lakshmikantham, "Multiple solutions of two-point boundary value problems of ordinary differential equations in Banach spaces," Journal of Mathematical Analysis and Applications, vol. 129, no. 1, pp. 211-222, 1988.

[5] B. Liu, "Positive solutions of a nonlinear four-point boundary value problems in Banach spaces," Journal of Mathematical Analysis and Applications, vol. 305, no. 1, pp. 253-276, 2005.

[6] L. Liu, Z. Liu, and Y. Wu, "Infinite boundary value problems for nth-order nonlinear impulsive integro- differential equations in Banach spaces," Nonlinear Analysis. Theory, Methods and Applications A, vol. 67, no. 9, pp. 2670-2679, 2007.

[7] D. Guo, "Existence of positive solutions for nth-order nonlinear impulsive singular integrodifferential equations in Banach spaces," Nonlinear Analysis. Theory, Methods and Applications A, vol. 68, no. 9, pp. 2727-2740, 2008.

[8] Y. Zhao, H. Chen, and C. Xu, "Existence of multiple solutions for three-point boundary-value problems on infinite intervals in Banach spaces," Electronic Journal of Differential Equations, vol. 2012, no. 44, pp. 1-11, 2012. 
[9] X. Zhang, M. Feng, and W. Ge, "Existence and nonexistence of positive solutions for a class of nthorder three-point boundary value problems in Banach spaces," Nonlinear Analysis. Theory, Methods and Applications A, vol. 70, no. 2, pp. 584-597, 2009.

[10] Y.-L. Zhao and H.-B. Chen, "Existence of multiple positive solutions for m-point boundary value problems in Banach spaces," Journal of Computational and Applied Mathematics, vol. 215, no. 1, pp. 7990, 2008.

[11] W. Jiang and J. Zhang, "Positive solutions for $(k ; n-k)$ conjugate boundary value problems in Banach spaces," Nonlinear Analysis, Theory, Methods and Applications, vol. 71, no. 3-4, pp. 723-729, 2009.

[12] Y. Zhao and H. Chen, "Triple positive solutions for nonlinear boundary value problems in Banach space," Computers and Mathematics with Applications, vol. 58, no. 9, pp. 1780-1787, 2009.

[13] H. Chen and P. Li, "Existence of solutions of three-point boundary value problems in Banach spaces," Mathematical and Computer Modelling, vol. 49, no. 3-4, pp. 780-788, 2009.

[14] P. W. Eloe and B. Ahmad, "Positive solutions of a nonlinear nth orderboundary value problem with nonlinear conditions," Applied Mathematics Letters, vol. 18, no. 5, pp. 521-527, 2005.

[15] P. W. Eloe and J. Henderson, "Singular nonlinear $(k ; n-k)$ conjugate boundary value problems," Journal of Differential Equations, vol. 133, no. 1, pp. 136-151, 1997.

[16] R. P. Agarwal and D. O'Regan, "Multiplicity results for singular conjugate, focal, and $(n-p)$ problems," Journal of Differential Equations, vol. 170, no. 1, pp. 142-156, 2001.

[17] R. P. Agarwal and D. O'Regan, "Right focal singular boundary value problems," Zeitschrift für Angewandte Mathematik und Mechanik, vol. 79, no. 6, pp. 363-373, 1999.

[18] R. P. Agarwal, Focal Boundary Value Problems for Differential and Difference Equations, Mathematics and its Applications, Kluwer, Dordrecht, The Netherlands, 1998.

[19] P. W. Eloe and J. Henderson, "Positive solutions and nonlinear multipoint conjugate eigenvalue problems," Electronic Journal of Differential Equations, vol. 1997, no. 3, pp. 1-11, 1997.

[20] L. Kong and J. Wang, "The Green's function for $(k ; n-k)$ conjugate boundary value problems and its applications," Journal of Mathematical Analysis and Applications, vol. 255, no. 2, pp. 404-422, 2001.

[21] G. W. Zhang and J. X. Sun, "Positive solutions of singular $(k ; n-k)$ Multi-point boundary value problems," Acta Mathematica Sinica, vol. 49, no. 2, pp. 391-398, 2006 (Chinese).

[22] K. Q. Lan, "Multiple positive solutions of conjugate boundary value problems with singularities," Applied Mathematics and Computation, vol. 147, no. 2, pp. 461-474, 2004.

[23] H. Su and Z. Wei, "Positive solutions to semipositone $(k ; n-k)$ conjugate eigenvalue problems," Nonlinear Analysis. Theory, Methods and Applications A, vol. 69, no. 9, pp. 3190-3201, 2008. 


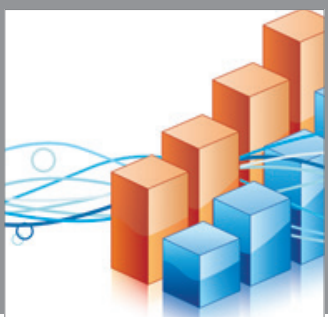

Advances in

Operations Research

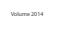

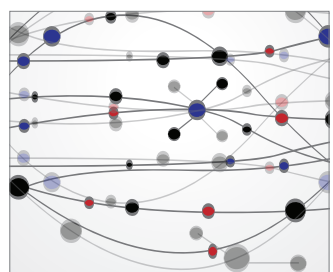

\section{The Scientific} World Journal
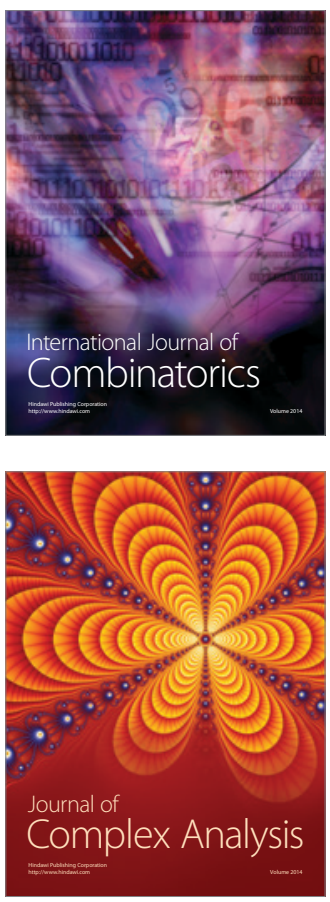

International Journal of

Mathematics and

Mathematical

Sciences
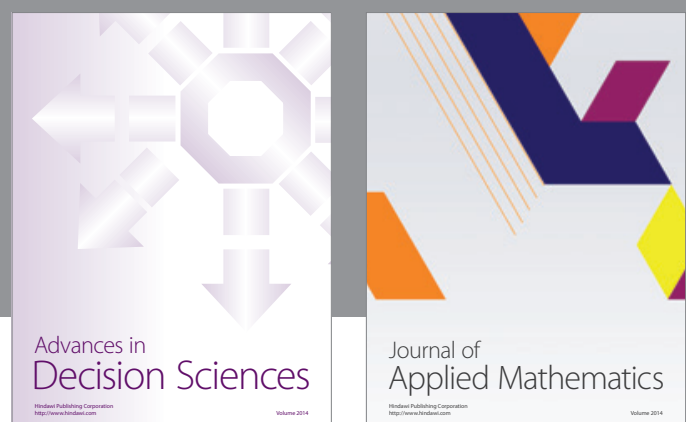

Journal of

Applied Mathematics
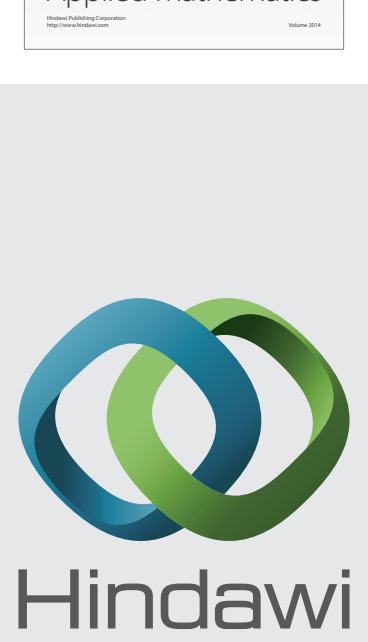

Submit your manuscripts at http://www.hindawi.com
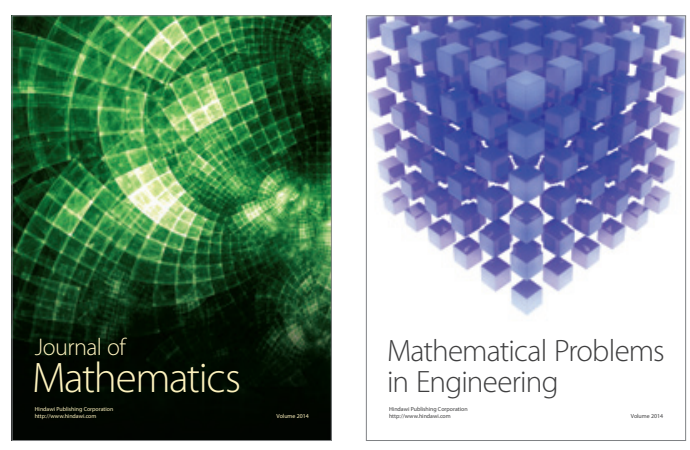

Mathematical Problems in Engineering
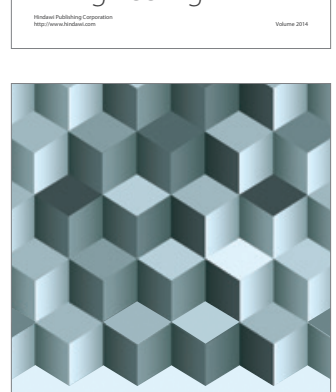

Journal of

Function Spaces
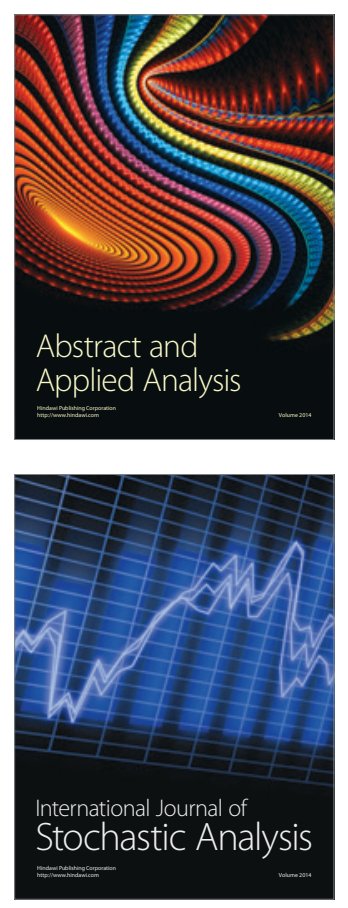

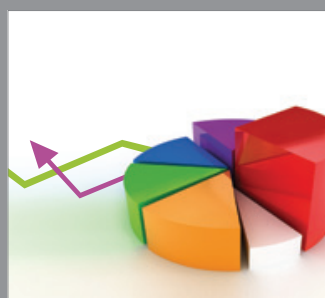

ournal of

Probability and Statistics

Promensencen
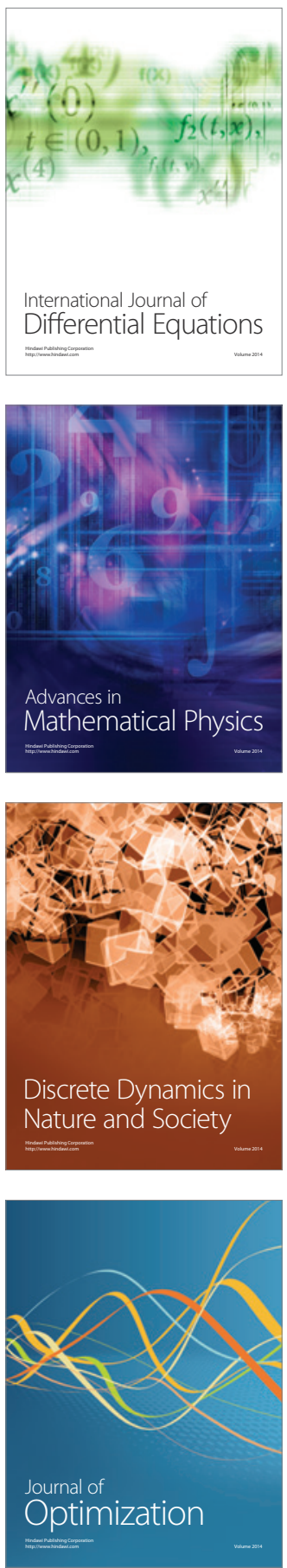